\title{
Determination of the Complete Elasticity of Nephila pilipes Spider Silk
}

\author{
Zuyuan Wang, Yu Cang, Friedrich Kremer, Edwin L. Thomas, and George Fytas* \\ Cite This: Biomacromolecules 2020, 21, 1179-1185 \\ Read Online
}

ABSTRACT: Spider silks are remarkable materials designed by nature to have extraordinary elasticity. Their elasticity, however, remains poorly understood, as typical stress-strain experiments only allow access to the axial Young's modulus. In this work, microBrillouin light spectroscopy (micro-BLS), a noncontact, nondestructive technique, is utilized to probe the direction-dependent phonon propagation in the Nephila pilipes spider silk and hence solve its full elasticity. To the best of our knowledge, this is the first demonstration on the determination of the anisotropic Young's moduli, shear moduli, and Poisson's ratios of a single spider fiber.

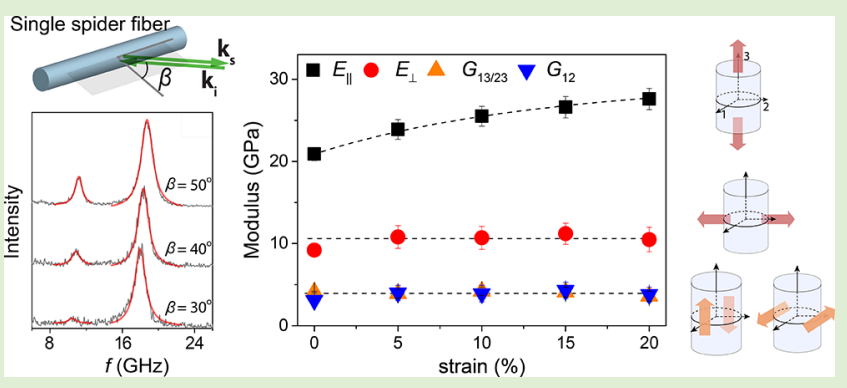
The axial and lateral Young's moduli are found to be $20.9 \pm 0.8$ and $9.2 \pm 0.3 \mathrm{GPa}$, respectively, and the anisotropy of the Young's moduli further increases upon stretching. In contrast, the shear moduli and Poisson's ratios exhibit very weak anisotropy and are robust to stretching.

\section{INTRODUCTION}

Spider silks are remarkable biomaterials with a delicate composition and hierarchical structure that give rise to their high tensile moduli. Among the seven types of silks in a typical spider web, the most intensively studied one is the major ampullate (MA) silk (also called the dragline silk), which serves as the scaffold of the web and the lifeline for spiders in danger. ${ }^{1}$ The outstanding elastic properties of the MA silk plus its biocompatibility have inspired many applications in fields like hunting, fishing, racket sports, bandages, bulletproof vests, and medical operations. ${ }^{2-4}$ So far, the studies on the composition of the MA silk have reached a consensus regarding the types and structures of silk proteins, but regenerate silk fibers made from spider silk proteins have not yet achieved the same level of Young's modulus of the MA silk. $^{5-8}$ This implies that besides composition, the structural organization in the spider silk plays an important role. To understand the internal structure of the spider silk, one could use direct methods, including transmission electron microscopy (TEM), ${ }^{9}$ NMR, ${ }^{10,11}$ X-ray scattering, ${ }^{7,12-14}$ Fourier transform infrared (FTIR), ${ }^{15,16}$ and atomic force microscopy (AFM) imaging, ${ }^{14,17}$ or indirect methods, such as stressstrain $^{6,18-36}$ and AFM-based ${ }^{31,37}$ mechanical deformation measurements. Based on the direct methods, it has been shown that spider silk is composed of thin skin and many densely packed nanofibrils oriented along the fiber axis. ${ }^{8,9,12}$ The nanofibrils comprise crystalline and amorphous parts with a crystallinity around $22 \%$ and featuring nanoconfinement (i.e., the crystallites are a few nanometers in each dimension). ${ }^{8,12,30,38}$ The lack of long-range order, however, renders the direct methods inapplicable for gaining the full structural information. The idea behind the more versatile indirect methods (e.g., the stress-strain, AFM three-point bending experiments) originates from the structure-property relations. Based on such experiments, the axial Young's modulus was obtained and a structural model was proposed to explain the strain-hardening/strain-weakening behaviors of spider silks. ${ }^{30}$ However, despite all the efforts, a satisfactory understanding of the structure of MA silk is still lacking. To gain a more complete understanding, additional elastic properties beyond the axial Young's modulus available from typical stress-strain measurements are needed. In addition to the local structure relation, the high-frequency (elastic) moduli are related to the intermolecular potential of the system. ${ }^{39}$

As a noncontact, nondestructive technique, Brillouin light spectroscopy (BLS) has been utilized to determine the elastic stiffness tensor of biomaterials, including protein, ${ }^{40}$ collagen, ${ }^{41}$ and muscle ${ }^{42}$ fibers. In the case of spider silk, there are two publications $^{43,44}$ on the elastic properties studied by BLS. However, both of them dealt with bundles of spider silks and involved misassignments of phonon modes in the BLS spectra, as detailed in Section S1 and also revealed by our analysis. Briefly, the mistaken phonon mode assignments in ref 43 resulted in unphysical elastic properties of spider silks (e.g., extremely large elastic constants, negative Poisson's ratios, etc.), whereas the erroneous mode assignments in ref 44 led to

Received: November 22, 2019

Revised: December 27, 2019

Published: January 14, 2020 
a misclaimed phononic band gap in spider silk. In this work, we demonstrate the determination of the complete elastic properties of a single spider silk fiber by BLS measurements and examine the strain effect on the silk fiber's elastic properties.

\section{MATERIALS AND METHODS}

2.1. Silk Sample. This study focuses on the MA silk of the Nephila pilipes spider. The silk was collected by forcibly silking a restrained spider at a rate of $1 \mathrm{~cm} \mathrm{~s}^{-1}$. ${ }^{45}$ The spider silk was stored and measured under ambient conditions (e.g., temperature: $\sim 21{ }^{\circ} \mathrm{C}$, pressure: $\sim 1$ atm, relative humidity: $\sim 45 \%)$.

2.2. Brillouin Light Spectroscopy (BLS). BLS is a noninvasive technique based on the inelastic scattering of light by thermally excited hypersonic phonons. We used a green laser $\left(\lambda_{0}=532 \mathrm{~nm}\right.$ in air) with an input power of around $5 \mathrm{~mW}$ and a focal spot size of 2.0 $\pm 0.5 \mu \mathrm{m}$ as the incident light. The scattered light was detected by a six-path tandem Fabry-Perot interferometer (JRS Instruments). The probed phonon has a wave vector, $\mathbf{q}=\mathbf{k}_{\mathrm{s}}-\mathbf{k}_{\mathrm{i}}$, where $\mathbf{k}_{\mathrm{i}}$ and $\mathbf{k}_{\mathrm{s}}$ are the wave vectors of the incident and scattered light, respectively. The polarization of the incident and scattered light was selected to be either vertical $(\mathrm{V})$ or horizontal $(\mathrm{H})$ to the scattering plane defined by $\mathbf{k}_{\mathrm{i}}$ and $\mathbf{k}_{\mathrm{s}}$. All of the measurements were conducted on single spider fibers, which were cut from the same strand. We conducted the measurements in the transmission, reflection, and backscattering geometries to access phonons propagating in different directions. The frequency shifts from the BLS measurements were used to calculate the direction-dependent sound velocities, which were subsequently utilized to calculate the stiffness elastic constants and mechanical moduli. More details about the experiments and data analysis are available in Sections S2-S7.

\section{RESULTS AND DISCUSSION}

3.1. Features of Elasticity Anisotropy. Before presenting the BLS measurement results, we illustrate the structure and the elastic anisotropy of the spider silk. Figure 1a-c shows the hierarchical structure of the spider silk. A typical spider web includes MA silks as the scaffold, each MA silk consists of many smaller fibrils, and each fibril contains crystalline and amorphous parts. The diameter of the single fiber is $8.3 \pm 0.6$ $\mu \mathrm{m}$, as determined from the scanning electron microscopy (SEM) image in Figure 1d. To illustrate the anisotropic elasticity of the spider silk, we show a schematic polar plot of the Young's and shear moduli in Figure 1e and the meanings of four characteristic moduli in Figure 1f. The variation of the moduli with $\alpha$, an angle with respect to the fiber axis, is a prominent feature of the mechanical anisotropy.

3.2. Anisotropic Elastic Moduli. To determine the mechanical properties, knowledge of the elastic stiffness tensor is a prerequisite. It is well-known that elastic stiffness constants are coupled with sound velocities in the framework of the Christoffel's equation. ${ }^{46-49}$ Given the direction-dependent sound velocities, the elastic constants can be determined via nonlinear $\chi^{2}$ fitting. ${ }^{50}$ Whereas the elastic tensor (in the Voigt notation) of a general anisotropic elastic material involves 21 independent elastic constants, the number could be significantly reduced by considering the structural symmetry. For spider silk, a natural choice is a transversely isotropic model, ${ }^{41,43}$ which leads to an elastic tensor with 5 independent constants (e.g., $C_{11}, C_{13}, C_{33}, C_{44}, C_{66}$ ) (eq S1). We note that the possible existence of nanopores and weak fibrillar interfaces in the spider silk ${ }^{9,51}$ affects neither the assumption of the transverse isotropy nor the BLS measurement results because BLS is only sensitive to density variations on the length scale of a few hundred nanometers. To facilitate the analysis, we
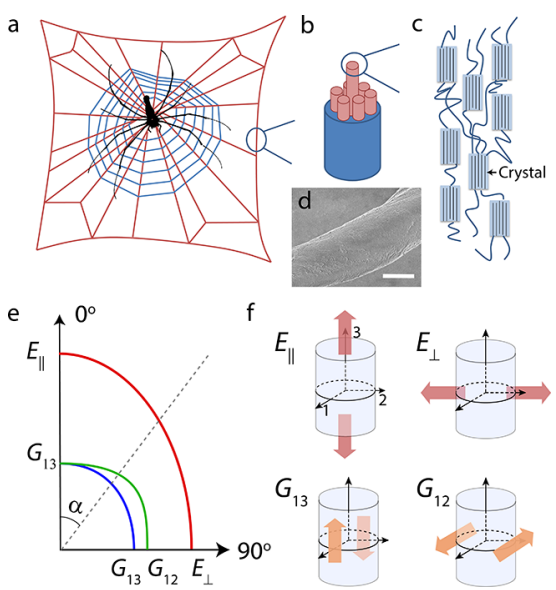

Figure 1. Hierarchical structure and anisotropic elasticity of spider fiber. (a) Schematic of a N. pilipes spider orb web. The red and blue lines denote the major ampullate (MA) and flagelliform silks, respectively. (b) Illustration of the skin-core structure of the MA spider silk. The red rods represent the fibrils in the core. (c) The fibrils comprise packed nanocrystals interconnected by amorphous chains. (d) SEM image of the MA spider silk measured in this work. The scale bar is $5 \mu \mathrm{m}$. (e) Schematic polar diagram of Young's moduli, $E$ (red line) and shear moduli, $G$ (blue and green lines), as a function of the angle $(\alpha)$ relative to the fiber axis. $E_{\|}\left(G_{13}\right)$ and $E_{\perp}$ $\left(G_{12}\right)$ represent the Young's (shear) moduli parallel and normal to the fiber axis, respectively. (f) Illustrations of the meanings of the elastic properties in (e). The red and orange arrows indicate the directions of the exerted stresses.

constructed a " 123 " coordinate system with the " 3 "-axis being parallel to the fiber axis (Figure 1f). The transverse isotropy assumption allowed us to restrict the consideration of phonon propagation directions in the "23" plane with $\alpha$, the angle between the phonon wave vector and the fiber axis, in the range of $0-90^{\circ}$ (Section S2). For each propagation direction, there exists one quasi-longitudinal (Q-L), one quasi-transverse (Q-T), and one pure-transverse (P-T) phonon mode. By probing the sound velocities of the three modes at multiple $\alpha$ angles, the elastic tensor and hence the full elasticity could be determined.

To obtain the direction-dependent sound velocities, we employed micro-BLS with a focal spot size of $2.0 \pm 0.5 \mu \mathrm{m}$, which enabled measurements on a single spider fiber. BLS experiments on single fibers eliminate the possible multiphonon-scattering processes and broadening of the probed phonon wave vector that could distort the spectra of a bundle of fibers, although the experiments take comparatively longer because of the small scattering volume. We conducted measurements in the transmission, reflection, and backscattering geometries (Figure 2) to probe the phonon propagation directions parallel $\left(\alpha=0^{\circ}\right)$, normal $\left(\alpha=90^{\circ}\right)$, and oblique $\left(0^{\circ}<\alpha<90^{\circ}\right)$ to the fiber axis. For a given $\alpha$, the existing Q-L, Q-T, and P-T were selectively probed by different polarization configurations (e.g., $\mathrm{VV}, \mathrm{VH}, \mathrm{HH}$ ) of the incident and scattered light beams. In theory, Q-L and Q-T modes are detectable in $\mathrm{VV}$ and $\mathrm{HH}$ polarization configurations, whereas P-T modes are observable in $\mathrm{VH}$ and $\mathrm{HV}$ polarization configurations (Section S7 and Table S4).

For practical intensity considerations, we selectively conducted the transmission and reflection measurements using $\mathrm{VV}$ and $\mathrm{VH}$ polarization configurations and the backscattering measurements using $\mathrm{HH}$ and $\mathrm{VH}$ polarization 


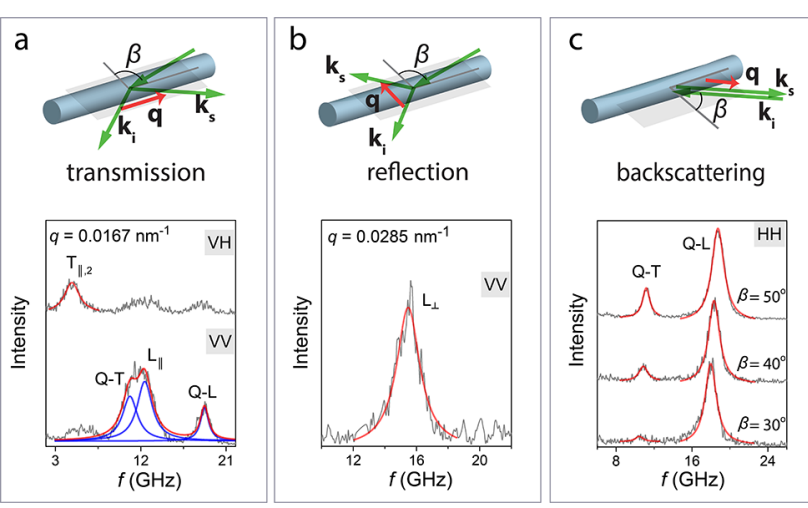

Figure 2. Hypersonic phonons in single spider fiber resolved by micro-BLS in three scattering geometries. The exemplary BLS spectra are recorded in the (a) transmission, (b) reflection, and (c) backscattering geometries. In the schematics, the rod represents a single N. pilipes MA spider fiber; $\mathbf{k}_{\mathrm{i}}$ and $\mathbf{k}_{\mathrm{s}}$, being the wave vectors of the incident and scattered light inside the fiber, respectively, define the scattering plane (gray plane); $\beta$ is the incident angle of the laser beam outside the fiber; and the red arrow denotes the phonon wave vector, $\mathbf{q}=\mathbf{k}_{\mathrm{s}}-\mathbf{k}_{\mathrm{i}}$. The transmission, reflection, and backscattering geometries feature phonon wave vectors parallel, normal, and oblique to the fiber axis, respectively. $\mathrm{VV}, \mathrm{VH}$, and $\mathrm{HH}$ denote different polarization configurations of the incident and scattered light beams, with $\mathrm{V}(\mathrm{H})$ representing the polarization vertical (horizontal) to the scattering plane. In the transmission geometry, a typical VV spectrum includes three phonon modes $\left(\mathrm{Q}-\mathrm{T}, \mathrm{L}_{\|}, \mathrm{Q}-\mathrm{L}\right)$, whereas a typical $\mathrm{VH}$ spectrum gives one phonon mode $\left(\mathrm{T}_{\|}\right)$. In the reflection geometry, a typical VV spectrum shows one phonon mode $\left(\mathrm{L}_{\perp}\right)$. In the backscattering geometry, a typical $\mathrm{HH}$ spectrum exhibits two phonon modes (Q-T, Q-L). The experimental BLS spectra (gray lines) recorded at different $q$ values are well-represented by Lorentzian shapes (red and blue lines). configurations. For simplicity, the Q-L $\left(0^{\circ}\right)$, Q-T $\left(0^{\circ}\right)$, P-T $\left(0^{\circ}\right)$, Q-L $\left(90^{\circ}\right)$, Q-T $\left(90^{\circ}\right)$, P-T $\left(90^{\circ}\right)$, Q-L $\left(0^{\circ}<\alpha<90^{\circ}\right)$, Q-T $\left(0^{\circ}<\alpha<90^{\circ}\right)$, and $\mathrm{P}-\mathrm{T}\left(0^{\circ}<\alpha<90^{\circ}\right)$ phonon modes are denoted in the following as $\mathrm{L}_{\|}, \mathrm{T}_{\|, 1}, \mathrm{~T}_{\|, 2}, \mathrm{~L}_{\perp}, \mathrm{T}_{\perp, 1}, \mathrm{~T}_{\perp, 2}, \mathrm{Q}-\mathrm{L}$, $\mathrm{Q}-\mathrm{T}$, and P-T, respectively. We note that the three scattering geometries (i.e., transmission, reflection, backscattering) and three polarization configurations (i.e., $\mathrm{VV}, \mathrm{HH}, \mathrm{VH}$ ) adopted in this work give a complete set of BLS experiments necessary for characterizing transversely anisotropic materials.

Figure 2a shows exemplary $\mathrm{VV}$ and $\mathrm{VH}$ BLS spectra recorded in the transmission geometry, where $\mathbf{q}$ is parallel to the fiber axis. The strong low-frequency peak in the $\mathrm{VH}$ spectrum corresponds to a $T_{\|, 2}$ mode, whereas the $\mathrm{VV}$ spectrum includes contributions from an $\mathrm{L}_{\|}$, a $\mathrm{Q}-\mathrm{T}$, and a $\mathrm{Q}$ $\mathrm{L}$ mode. The appearance of the last two modes (Q-T and Q-L) results from the artificial backscattering in transmission measurements (Figures S1 and S4). ${ }^{49,52-54}$ Figure $2 \mathrm{~b}$ presents a typical VV BLS spectrum obtained in the reflection geometry with $\mathbf{q}$ normal to the fiber axis. The single peak is assigned to an $\mathrm{L}_{\perp}$ mode, i.e., a longitudinal phonon propagating normal to the fiber axis. The expected $\mathrm{T}_{\perp, 2}$ modes in the $\mathrm{VH}$ spectra were not observed, possibly due to their weak intensity at high scattering angles (Section S7). Figure 2c displays representative $\mathrm{HH}$ BLS spectra probed in the backscattering geometry at incident angles, $\beta=30,40$, and $50^{\circ}$ (see Figure $S 3$ for the full spectra), where $\mathbf{q}$ is along the scattered light and the corresponding $\alpha$ is in the range of $45-90^{\circ}$. The two peaks are assigned to Q-T and Q-L modes, and their frequencies vary with $\beta$. The P-T modes observable in the backscattering $\mathrm{VH}$ spectra were detected only at intermediate incident angles; an example of the VH spectrum is shown in Figure S6.

The phonon wave vectors and frequency shifts from the BLS spectra were used to calculate the sound velocities as $v=2 \pi f / q$. To calculate the magnitude of the phonon wave vector in the reflection and backscattering measurements, the refractive
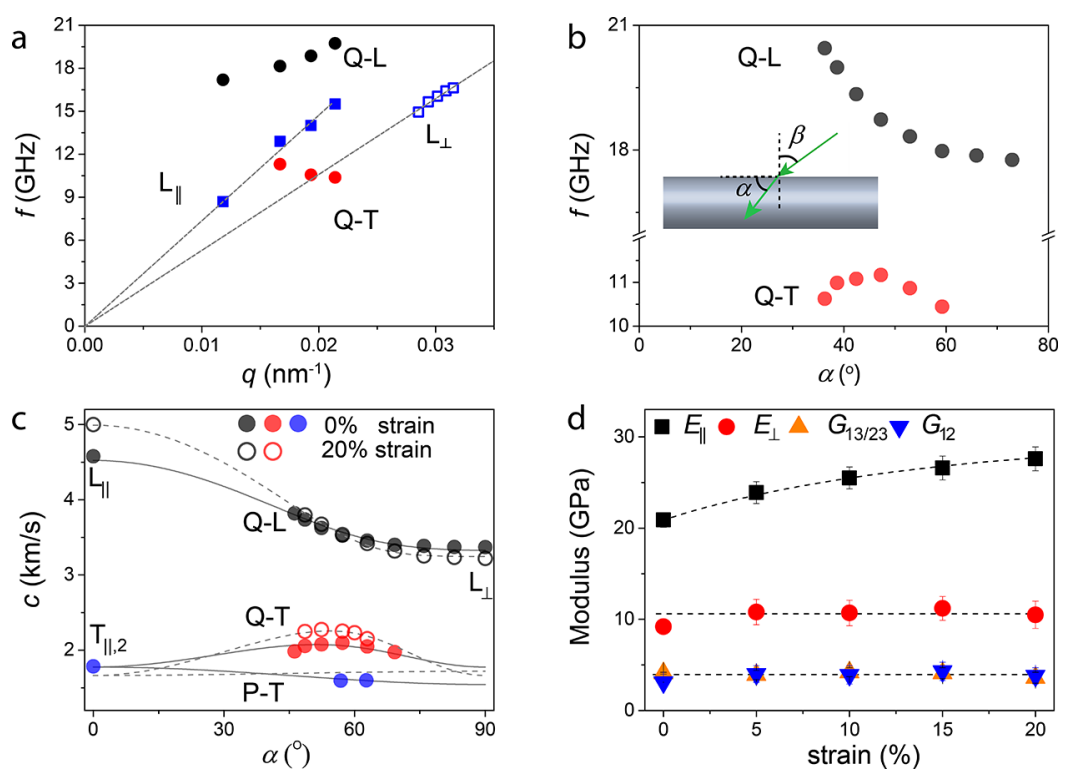

Figure 3. Phonon dispersions in single spider fiber and strain effect on the elastic properties. (a) Dispersion relations measured in the transmission (solid symbols) and reflection (open symbols) geometries. The $\mathrm{L}_{\|}, \mathrm{L}_{\perp}, \mathrm{Q}-\mathrm{L}$, and $\mathrm{Q}-\mathrm{T}$ modes are denoted by different colors. (b) Angle-dependent phonon frequencies from backscattering measurements. (c) Angle-dependent sound velocities in the native ( $0 \%$ strain) and stretched (20\% strain) single spider fibers. The lines represent theoretical predictions by eqs $S 2-S 4$. (d) Variation of the axial and lateral Young's moduli $\left(E_{\|}, E_{\perp}\right)$ and shear moduli $\left(G_{13 / 23}, G_{12}\right)$ with the applied strain. The error bars are calculated according to principles of uncertainty propagation (Section S6). Error bars smaller than the symbol size are not shown. 
index of the spider silk is required. Since the spider silk was observed to be birefringent by polarized optical microscopy measurements (inset to Figure S2), VV and $\mathrm{HH}$ backscattering BLS spectra were resolved at $\beta=0^{\circ}$ to quantify the optical anisotropy (Section S4). The principal refractive indices were determined to be $n_{\mathrm{o}}=1.40 \pm 0.01$ and $n_{\mathrm{e}}=1.46 \pm 0.02$. This optical birefringence was taken into account while calculating the $q$ and $\alpha$ for all the phonon modes (Section S3).

To verify the acoustic nature of the observed phonons, angle-resolved VV measurements were performed in the transmission and reflection geometries by varying the incident angle, $\beta$. In these measurements, the magnitude of the phonon wave vector varies, whereas its direction remains unchanged. In Figure 3a, two linear dispersions are observed, which correspond to the $\mathrm{L}_{\|}$and $\mathrm{L}_{\perp}$ modes observed in the transmission and reflection geometries, respectively. In contrast, the Q-T and Q-L modes observed in the transmission geometry show nonlinear phonon dispersions (Figure 3a), assuming that they have the same $\mathbf{q}$ with $\mathrm{L}_{\|}$. This suggests that the Q-T and Q-L modes might originate from artificial backscattering. ${ }^{49,52-54}$ To verify this hypothesis, we compared their frequencies with those of the $\mathrm{Q}-\mathrm{T}$ and $\mathrm{Q}-\mathrm{L}$ modes from the real backscattering experiments at commensurate incident angles. Notably, the hypothesis is confirmed by the perfect agreement of the phonon frequencies from the two scattering geometries (Figure S4). Therefore, the frequencies of the Q-T and Q-L modes in the BLS spectra recorded in the transmission geometry should be plotted with their actual $q$ values in the phonon dispersion diagram. The angle-resolved (or $q$-dependent) measurements allow unambiguous assignments of the acoustic phonons and thereby access to their correct sound velocities. Figure $3 \mathrm{~b}$ shows the variation of the frequencies of the Q-L and Q-T modes obtained from the backscattering measurements as a function of $\alpha$. The dispersive Q-L and Q-T modes imply a mechanical anisotropy of the silk fiber.

Figure $3 \mathrm{c}$ depicts the direction-dependent sound velocities of the spider fiber at $0 \%$ strain (i.e., the native state). We observed the Q-L and Q-T phonon modes at multiple $\alpha$ values, whereas the P-T mode was observed only at $\alpha=0,40$, and $50^{\circ}$ for the spider fiber at $0 \%$ strain, similar to the result in a previous study on dry rat-tail tendon collagen by Cusack and Miller. ${ }^{41}$ It is worth mentioning that the present work reports for the first time the sound velocities of the Q-L, Q-T, and P-T modes in multiple propagation directions in a single spider fiber. At $0 \%$ strain, as $\alpha$ increases from 0 to $90^{\circ}, v_{\mathrm{Q}-\mathrm{L}}$ decreases from $4580 \pm 90$ to $3370 \pm 70 \mathrm{~m} \mathrm{~s}^{-1}, v_{\mathrm{P}-\mathrm{T}}$ shows a slight decrease from $1790 \pm 40$ to $1600 \pm 30 \mathrm{~m} \mathrm{~s}^{-1}$, whereas $v_{\mathrm{Q}-\mathrm{T}}$ first increases, achieves a maximum at around $\alpha=50^{\circ}$, and then decreases. Based on the $v_{\mathrm{Q}-\mathrm{L}}(\alpha), v_{\mathrm{Q}-\mathrm{T}}(\alpha)$, and $v_{\mathrm{P}-\mathrm{T}}(\alpha)$ data, we determined the five independent elastic stiffness constants (i.e., $C_{11}, C_{13}, C_{33}, C_{44}, C_{66}$ ) via nonlinear $\chi^{2}$ fitting (Section S5). The density of the spider fiber was assumed to be $1300 \mathrm{~kg} \mathrm{~m}^{-3}$, as indicated in previous studies. ${ }^{55-57}$ The best fits to the experimental sound velocities at $0 \%$ strain are shown in Figure $3 \mathrm{c}$ as solid lines, with the residuals of the fitted sound velocities shown in Figure S5. From the obtained elastic stiffness constants (Table S1), we calculated the characteristic mechanical properties, including the axial and lateral Young's moduli $\left(E_{\|}, E_{\perp}\right)$, shear moduli $\left(G_{13}=G_{23}, G_{12}\right)$, and Poisson's ratios $\left(\nu_{31}=\nu_{32}, \nu_{12}\right)$ (only five of them are independent), as summarized in Table S3. The relevant formulas and physical meanings of the mechanical properties are presented in Table S2.

We also conducted the same measurements on spider fibers at stretching strains in the range of $5-20 \%$. Note that the spider fiber broke at a strain of around $22 \%$. The directiondependent sound velocities in the spider fiber at a $20 \%$ strain are shown in Figure 3c. For the stretched spider fibers, no unambiguous P-T mode was observed, which could be attributed to the vanishing scattering intensity governed by the Pockels (elasto-optic) coefficients (Section S7 and Table S4) and inhibited a unique determination of $C_{66}$. To complete the elastic tensor determination, we assumed the Poisson's ratio $\nu_{31}$ to be equal to that at $0 \%$ strain. The best fits to the experimental sound velocities at $20 \%$ strain are shown in Figure $3 \mathrm{c}$ as dashed lines. At $20 \%$ strain, the $v\left(\mathrm{~L}_{\|}\right)$and $v\left(\mathrm{~L}_{\perp}\right)$ are higher and lower than those at $0 \%$ strain, respectively, implying a stronger elasticity anisotropy. Similar angledependent sound velocities in the spider fibers at 5, 10, and $15 \%$ strains are shown in Figure S7. The elastic stiffness constants and characteristic mechanical properties of the spider fiber at different strains are summarized in Tables S1 and S3, respectively.

Figure $3 \mathrm{~d}$ shows the strain dependence of $E_{\|}, E_{\perp}, G_{13 / 23}$, and $G_{12}$. As the strain increases from 0 to $20 \%, E_{\|}$increases from $20.9 \pm 0.8$ to $27.6 \pm 1.3 \mathrm{GPa}$, confirming a strain-stiffening behavior of the silk fiber upon stretching. In contrast, $E_{\perp}, G_{13}$, and $G_{12}$ are quite robust to the stretching strain and have values around $10.8 \pm 1.4,4.0 \pm 0.6$, and $4.0 \pm 1.0 \mathrm{GPa}$, respectively. The corresponding mechanical anisotropy ratio, $E_{\|} / E_{\perp}$, increases from 2.3 to 2.6. The two Poisson's ratios of the spider fiber, $\nu_{31}$ and $\nu_{12}$, possess typical values of polymers $(\sim 0.33)^{58,59}$ and have a weak dependence on the applied strain (Table S3).

3.3. Comparison with Literature Results. To compare the elastic properties of the spider silk from this work with the literature data, we summarize the experimental elastic moduli of the MA silk of four types of Nephila spiders in Figure 4. A more comprehensive summary of the literature data on the elastic properties of spider silks is included in Table S5. The following points emerge from Figure 4. (i) Unlike typical

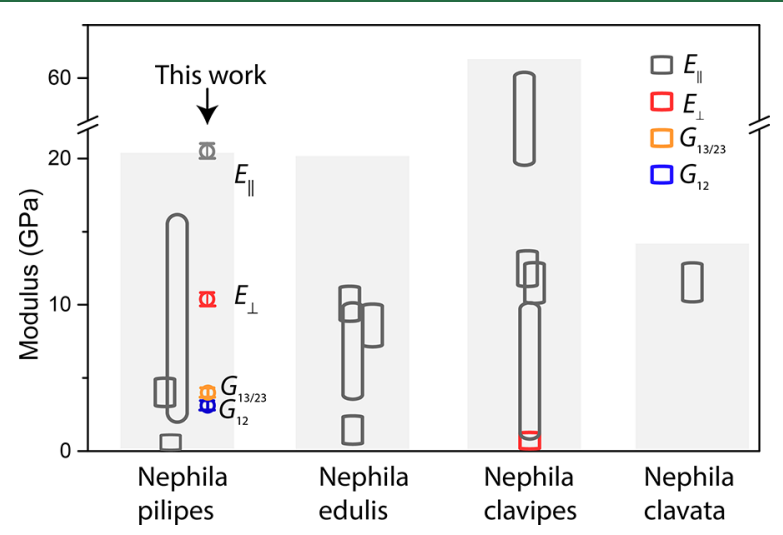

Figure 4. Experimental elastic moduli of the MA silk of four types of Nephila spiders. The open and solid symbols represent data from previous work and this work, respectively. The axial and lateral Young's moduli $\left(E_{\|}, E_{\perp}\right)$ and shear moduli $\left(G_{13 / 23}, G_{12}\right)$ are represented by blue, red, and green symbols, respectively. The height of the symbols denotes the range of experimental data in each work. N. pilipes: refs 14, 33, 36; N. edulis: refs 20-23; N. clavipes: refs 19, 25, 26, 63; and N. clavata: ref 24. 
stress-strain experiments that only allow the determination of $E_{\|}$, BLS enables unique access to the complete elastic moduli. (ii) Compared to the typically widespread $E_{\|}$data from the stress-strain measurements, $E_{\|}$determined by BLS falls within a much narrower range. This could be attributed to the noninvasive nature of BLS and thus its insensitiveness to sample defects. (iii) Previous studies based on stress-strain experiments reported $E_{\|}$of the $N$. pilipes MA silk in the range of $0-16 \mathrm{GPa}{ }^{14,32,35}$ The comparatively larger elastic $E_{\|}(20.9$ $\pm 0.8 \mathrm{GPa}$ ) in the present work might be related to the absence of dissipation at the $\mathrm{GHz}$ frequencies in BLS measurements. $^{41}$ (iv) The mechanical anisotropy $\left(E_{\|} / E_{\perp}=\right.$ 2.3-2.6) suggests a predominantly axial orientation of the crystalline part in the spider fiber. ${ }^{8,9,12}$ Notably, an elastic anisotropy of 2.3 is achieved in the native N. pilipes MA silk with a low crystallinity of around $22 \%$. (v) Stress-strain experiments were also used to measure the lateral Young's modulus $\left(E_{\perp}\right)$ of the $N$. clavipes spider fiber. ${ }^{25}$ However, the influence of the substrate and the uncertainties in the crosssectional area and strain calculations render the data's reliability questionable. BLS measurements do not suffer from such limitations, although BLS is generally limited to transparent materials with weak absorption at the employed laser wavelength. We point out that BLS is capable of measuring the elastic properties on a scale commensurate with the wavelength of the probing phonon, which is around 200$400 \mathrm{~nm}$ for the measurements in this work. Since the diameter of the core (around $8 \mu \mathrm{m}$ ) is much larger than the phonon wavelengths, which are larger than the thickness of the skin (around $100 \mathrm{~nm}),{ }^{60}$ the measured elastic properties represent the effective properties of the entire spider fiber (i.e., core + shell) with the core being the dominant fraction. This is consistent with a recent paper by Yazawa et al., ${ }^{60}$ which reports that the mechanical properties of spider silk are mainly determined by the core, without significant changes upon removal of the skin. Recently, $\mathrm{Wu}$ et al. ${ }^{61}$ have reported that the elastic longitudinal modulus $M$ (on the order of GPa) of biological systems (e.g., cells, tissues, and hydrogels) measured by $\mathrm{BLS}$ at $\mathrm{GHz}$ frequencies may not be related to Young's modulus (on the order of $\mathrm{kPa}$ ) measured by rheology obtained at much low $(\mathrm{kHz})$ frequencies, thus criticizing the finding by Scarcelli et al. ${ }^{62}$ The justified criticism is based on the viscoelastic nature of biomaterials, for which the Young's moduli depend on the cross-linking density of the network. The only common property of the two moduli $(M$ and $G)$ is the local packing manifested by the glass transition temperature of the system.

Since the elastic properties of spider silk are strongly influenced by the species and physical condition of the spider, the procedure of collecting silk samples, the setup and procedure of measurements, and the laboratory ambient conditions (e.g., temperature, relative humidity), ${ }^{14,19-21,24,26,29,32,63}$ additional studies are needed to explore a larger sample space to draw a comprehensive conclusion on the elasticity of spider silk. Nevertheless, by resolving the complete elastic tensor of spider silk based on single-fiber measurements, the present work represents a significant step toward this goal. The elastic properties reported here could be used to guide and validate computer simulations, which could potentially further advance our understanding of the structure-property relation in spider silk. ${ }^{64}$ Moreover, the rigorous method presented in this work, combining micro-BLS measurements and analysis based on a continuum-mechanics-based model, could be extended to the studies of the elastic properties of other mechanically anisotropic materials (e.g., liquid crystals, silkworm silk, fiberlike structures, and fiber-reinforced composites).

\section{CONCLUSIONS}

We have studied the elastic properties of the $N$. pilipes MA silk by using micro-BLS. We demonstrate, for the first time, BLS measurements on a single spider fiber. The comprehensive multiangle measurements, careful consideration of the optical birefringence, and correct phonon mode assignments allowed us to determine the complete elastic tensor and characteristic mechanical properties of the spider silk. In the native state, the axial and lateral Young's moduli of the spider fiber are $20.9 \pm$ 0.8 and $9.2 \pm 0.3 \mathrm{GPa}$, respectively. Upon stretching by $20 \%$, the axial Young's modulus of the spider silk increases by $32 \%$, exhibiting a strain-stiffening behavior, whereas the lateral Young's moduli, shear moduli, and Poisson's ratios show weak strain dependences. The complete elastic properties and their strain dependences could be used to guide future simulations, allowing further elucidation of the structure-property relation in spider silk.

\section{ASSOCIATED CONTENT}

\section{sI Supporting Information}

The Supporting Information is available free of charge at https://pubs.acs.org/doi/10.1021/acs.biomac.9b01607.

Mistakes in two previous BLS studies on the elastic properties of spider silk, transversely isotropic elasticity model, BLS experiments, characterization of optical birefringence, $\chi^{2}$ fitting, uncertainty analysis, scattering intensity calculations, typical BLS spectra, explanation of artificial backscattering modes in transmission spectra, elastic constants, and literature data on axial Young's moduli of spider silks (PDF)

\section{AUTHOR INFORMATION}

\section{Corresponding Author}

George Fytas - Max Planck Institute for Polymer Research, 55128 Mainz, Germany; Institute of Electronic Structure and Laser, F.O.R.T.H, 70013 Heraklion, Greece; Phone: +49 6131-379-718; Email: fytas@mpip-mainz.mpg.de

\section{Authors}

Zuyuan Wang - Max Planck Institute for Polymer Research, 55128 Mainz, Germany; 이이.org/0000-0002-3174-4227

Yu Cang - Max Planck Institute for Polymer Research, 55128 Mainz, Germany

Friedrich Kremer - Institute of Experimental Physics I, University of Leipzig, 04103 Leipzig, Germany

Edwin L. Thomas - Department of Materials Science and Nano-Engineering, Rice University, Houston, Texas 77030, United States; O orcid.org/0000-0001-5911-6524

Complete contact information is available at:

https://pubs.acs.org/10.1021/acs.biomac.9b01607

\section{Author Contributions}

Z.W. and Y.C. contributed equally to this work. The manuscript was written through contributions of all of the authors. All of the authors have given approval to the final version of the manuscript. 


\section{Funding}

This work was financially supported by ERC AdG SmartPhon (Grant No. 694977).

\section{Notes}

The authors declare no competing financial interest.

\section{REFERENCES}

(1) Eisoldt, L.; Smith, A.; Scheibel, T. Decoding the secrets of spider silk. Mater. Today 2011, 14, 80-86.

(2) Scheibel, T. Spider silks: recombinant synthesis, assembly, spinning, and engineering of synthetic proteins. Microb. Cell Fact. 2004, 3, No. 14.

(3) Kluge, J. A.; Rabotyagova, O.; Leisk, G. G.; Kaplan, D. L. Spider silks and their applications. Trends Biotechnol. 2008, 26, 244-251.

(4) Teulé, F.; Miao, Y. G.; Sohn, B. H.; Kim, Y. S.; Hull, J. J.; Fraser, M. J.; Lewis, R. V.; Jarvis, D. L. Silkworms transformed with chimeric silkworm/spider silk genes spin composite silk fibers with improved mechanical properties. Proc. Natl. Acad. Sci. U.S.A. 2012, 109, 923928.

(5) Seidel, A.; Liivak, O.; Calve, S.; Adaska, J.; Ji, G.; Yang, Z.; Grubb, D.; Zax, D. B.; Jelinski, L. W. Regenerated spider silk: Processing, properties, and structure. Macromolecules 2000, 33, 775780.

(6) Heidebrecht, A.; Eisoldt, L.; Diehl, J.; Schmidt, A.; Geffers, M.; Lang, G.; Scheibel, T. Biomimetic fibers made of recombinant spidroins with the same toughness as natural spider silk. Adv. Mater. 2015, 27, 2189-2194.

(7) Anton, A. M.; Heidebrecht, A.; Mahmood, N.; Beiner, M.; Scheibel, T.; Kremer, F. Foundation of the outstanding toughness in biomimetic and natural spider silk. Biomacromolecules 2017, 18, 3954-3962.

(8) Giesa, T.; Buehler, M. J. Nanoconfinement and the Strength of Biopolymers. Annu. Rev. Biophys. 2013, 42, 651-673.

(9) Frische, S.; Maunsbach, A.; Vollrath, F. Elongate cavities and skin-core structure in Nephila spider silk observed by electron microscopy. J. Microsc. 1998, 189, 64-70.

(10) Yang, Z.; Liivak, O.; Seidel, A.; LaVerde, G.; Zax, D. B.; Jelinski, L. W. Supercontraction and backbone dynamics in spider silk: $13 \mathrm{C}$ and 2H NMR studies. J. Am. Chem. Soc. 2000, 122, 9019-9025.

(11) Creager, M. S.; Jenkins, J. E.; Thagard-Yeaman, L. A.; Brooks, A. E.; Jones, J. A.; Lewis, R. V.; Holland, G. P.; Yarger, J. L. Solid-state NMR comparison of various spiders' dragline silk fiber. Biomacromolecules 2010, 11, 2039-2043.

(12) Li, S. F.; McGhie, A. J.; Tang, S. L. New internal structure of spider dragline silk revealed by atomic force microscopy. Biophys. J. 1994, 66, 1209-1212.

(13) Yang, Z.; Grubb, D. T.; Jelinski, L. W. Small-angle X-ray scattering of spider dragline silk. Macromolecules 1997, 30, 82548261.

(14) Du, N.; Liu, X. Y.; Narayanan, J.; Li, L.; Lim, M. L. M.; Li, D. Design of superior spider silk: from nanostructure to mechanical properties. Biophys. J. 2006, 91, 4528-4535.

(15) Ling, S.; Qi, Z.; Knight, D. P.; Shao, Z.; Chen, X. Synchrotron FTIR microspectroscopy of single natural silk fibers. Biomacromolecules 2011, 12, 3344-3349.

(16) Anton, A. M.; Kossack, W.; Gutsche, C.; Figuli, R.; Papadopoulos, P.; Ebad-Allah, J.; Kuntscher, C.; Kremer, F. Pressure-dependent FTIR-spectroscopy on the counterbalance between external and internal constraints in spider silk of Nephila pilipes. Macromolecules 2013, 46, 4919-4923.

(17) Oroudjev, E.; Soares, J.; Arcidiacono, S.; Thompson, J. B.; Fossey, S. A.; Hansma, H. G. Segmented nanofibers of spider dragline silk: atomic force microscopy and single-molecule force spectroscopy. Proc. Natl. Acad. Sci. U.S.A. 2002, 99, 6460-6465.

(18) Calvert, P. D. Encyclopedia Materials Science and Engineering, Biological Macromolecules; Pergamon Press: Oxford, 1988; pp 334339.
(19) Cunniff, P. M.; Fossey, S. A.; Auerbach, M. A.; Song, J. W.; Kaplan, D. L.; Adams, W. W.; Eby, R. K.; Mahoney, D.; Vezie, D. L. Mechanical and thermal properties of dragline silk from the spider Nephila clavipes. Polym. Adv. Technol. 1994, 5, 401-410.

(20) Madsen, B.; Shao, Z. Z.; Vollrath, F. Variability in the mechanical properties of spider silks on three levels: interspecific, intraspecific and intraindividual. Int. J. Biol. Macromol. 1999, 24, 301306.

(21) Shao, Z.; Vollrath, F. The effect of solvents on the contraction and mechanical properties of spider silk. Polymer 1999, 40, 17991806.

(22) Madsen, B.; Vollrath, F. Mechanics and morphology of silk drawn from anesthetized spiders. Naturwissenschaften 2000, 87, 148153.

(23) Riekel, C.; Madsen, B.; Knight, D.; Vollrath, F. X-ray diffraction on spider silk during controlled extrusion under a synchrotron radiation X-ray beam. Biomacromolecules 2000, 1, 622-626.

(24) Osaki, S.; Ishikawa, R. Determination of elastic modulus of spider's silks. Polym. J. 2002, 34, 25.

(25) Ko, F. K. Natural Fibers, Plastics and Composites; Wallenberger, F. T.; Weston, N. E., Eds.; Springer: Boston, MA, 2004; pp 27-49.

(26) Elices, M.; Guinea, G. V.; Pérez-Rigueiro, J.; Plaza, G. R. Finding inspiration in Argiope trifasciata spider silk fibers. JOM 2005, $57,60-66$.

(27) Blackledge, T. A.; Hayashi, C. Y. Silken toolkits: biomechanics of silk fibers spun by the orb web spider Argiope argentata (Fabricius 1775). J. Exp. Biol. 2006, 209, 2452-2461.

(28) Swanson, B. O.; Anderson, S. P.; DiGiovine, C.; Ross, R. N.; Dorsey, J. P. The evolution of complex biomaterial performance: the case of spider silk. Integr. Comp. Biol. 2009, 49, 21-31.

(29) Boutry, C.; Řezáč, M.; Blackledge, T. A. Plasticity in major ampullate silk production in relation to spider phylogeny and ecology. PLoS One 2011, 6, No. e22467.

(30) Du, N.; Yang, Z.; Liu, X. Y.; Li, Y.; Xu, H. Y. Structural origin of the strain-hardening of spider silk. Adv. Funct. Mater. 2011, 21, 772778.

(31) Hansma, H. G. Biotechnology of Silk; Asakura, T.; Miller, T., Eds.; Biologically-Inspired Systems; Springer: Dordrecht, 2014; Vol. 5, pp 123-136.

(32) Madurga, R.; Blackledge, T. A.; Perea, B.; Plaza, G. R.; Riekel, C.; Burghammer, M.; Elices, M.; Guinea, G.; Pérez-Rigueiro, J. Persistence and variation in microstructural design during the evolution of spider silk. Sci. Rep. 2015, 5, No. 14820.

(33) Blamires, S. J.; Liao, C. P.; Chang, C. K.; Chuang, Y. C.; Wu, C. L.; Blackledge, T. A.; Sheu, H. S.; Tso, I. M. Mechanical performance of spider silk is robust to nutrient-mediated changes in protein composition. Biomacromolecules 2015, 16, 1218-1225.

(34) Madurga, R.; Plaza, G. R.; Blackledge, T. A.; Guinea, G. V.; Elices, M.; Pérez-Rigueiro, J. Material properties of evolutionary diverse spider silks described by variation in a single structural parameter. Sci. Rep. 2016, 6, No. 18991.

(35) Yarger, J. L.; Cherry, B. R.; Van Der Vaart, A. Uncovering the structure-function relationship in spider silk. Nat. Rev. Mater. 2018, 3, No. 18008 .

(36) Kerr, G. G.; Nahrung, H. F.; Wiegand, A.; Kristoffersen, J.; Killen, P.; Brown, C.; Macdonald, J. Mechanical properties of silk of the Australian golden orb weavers Nephila pilipes and Nephila plumipes. Biol. Open 2018, 7, No. bio029249.

(37) Hudson, S. D.; Zhurov, V.; Grbić, V.; Grbić, M.; Hutter, J. L. Measurement of the elastic modulus of spider mite silk fibers using atomic force microscopy. J. Appl. Phys. 2013, 113, No. 154307.

(38) Sun, M.; Zhang, Y.; Zhao, Y.; Shao, H.; Hu, X. The structureproperty relationships of artificial silk fabricated by dry-spinning process. J. Mater. Chem. 2012, 22, 18372-18379.

(39) Midya, J.; Cang, Y.; Egorov, S. A.; Matyjaszewski, K.; Bockstaller, M. R.; Nikoubashman, A.; Fytas, G. Disentangling the Role of Chain Conformation on the Mechanics of Polymer Tethered Particle Materials. Nano Lett. 2019, 19, 2715-2722. 
(40) Speziale, S.; Jiang, F.; Caylor, C. L.; Kriminski, S.; Zha, C. S.; Thorne, R. E.; Duffy, T. S. Sound velocity and elasticity of tetragonal lysozyme crystals by Brillouin spectroscopy. Biophys. J. 2003, 85, 3202-3213.

(41) Cusack, S.; Miller, A. Determination of the elastic constants of collagen by Brillouin light scattering. J. Mol. Biol. 1979, 135, 39-51.

(42) Berovic, N.; Thomas, N.; Thornhill, R. A.; Vaughan, J. M. Observation of Brillouin scattering from single muscle fibres. Eur. Biophys. J. 1989, 17, 69-74.

(43) Koski, K. J.; Akhenblit, P.; McKiernan, K.; Yarger, J. L. Noninvasive determination of the complete elastic moduli of spider silks. Nat. Mater. 2013, 12, 262.

(44) Schneider, D.; Gomopoulos, N.; Koh, C. Y.; Papadopoulos, P.; Kremer, F.; Thomas, E. L.; Fytas, G. Nonlinear control of highfrequency phonons in spider silk. Nat. Mater. 2016, 15, 1079.

(45) Work, R. W.; Emerson, P. D. An apparatus and technique for the forcible silking of spiders. J. Arachnol. 1982, 10, 1-10.

(46) Cheadle, S. P.; Brown, R. J.; Lawton, D. C. Orthorhombic anisotropy: A physical seismic modeling study. Geophysics 1991, 56, $1603-1613$.

(47) Mah, M.; Schmitt, D. R. Experimental determination of the elastic coefficients of an orthorhombic material. Geophysics 2001, 66, $1217-1225$.

(48) Ryu, M.; Cang, Y.; Wang, Z.; Fytas, G.; Morikawa, J. Temperature-Dependent Thermoelastic Anisotropy of the Phenyl Pyrimidine Liquid Crystal. J. Phys. Chem. C 2019, 123, 17148-17154. (49) Wang, Z.; Rolle, K.; Schilling, T.; Hummel, P.; Philipp, A.; Kopera, B. A.; Lechner, A. M.; Retsch, M.; Breu, J.; Fytas, G. Tunable thermoelastic anisotropy in hybrid Bragg stacks with extreme polymer confinement. Angew. Chem., Int. Ed. 2020, 59, 1286-1294.

(50) Zgonik, M.; Bernasconi, P.; Duelli, M.; Schlesser, R.; Günter, P.; Garrett, M. H.; Rytz, D.; Zhu, Y.; Wu, X. Dielectric, elastic, piezoelectric, electro-optic, and elasto-optic tensors of BaTiO 3 crystals. Phys. Rev. B 1994, 50, 5941.

(51) Zhang, W.; Ye, C.; Zheng, K.; Zhong, J.; Tang, Y.; Fan, Y.; Buehler, M. J.; Ling, S.; Kaplan, D. L. Tensan silk-inspired hierarchical fibers for smart textile applications. ACS Nano 2018, 12, 6968-6977.

(52) Krüger, J. K.; Bastian, H.; Asbach, G. I.; Pietralla, M. The elastic properties of C $36 \mathrm{H} 74$ single crystals from Brillouin-spectroscopy. Polym. Bull. 1980, 3, 633-640.

(53) Krüger, J.; Peetz, L.; Pietralla, M. Brillouin scattering of semicrystalline poly (4-methyl-1-pentene): study of surface effects of bulk and film material. Polymer 1978, 19, 1397-1404.

(54) Fujita, Y.; Kobayashi, H. Dispersion and attenuation on the Brillouin sound waves of a lubricant: Di (2-ethylhexyl) sebacate under high pressures. J. Appl. Phys. 2011, 110, No. 033538.

(55) Saravanan, D. Spider silk-structure, properties and spinning. J. Text. Apparel, Technol. Manage. 2006, 5, 1-20.

(56) Agnarsson, I.; Dhinojwala, A.; Sahni, V.; Blackledge, T. A. Spider silk as a novel high performance biomimetic muscle driven by humidity. J. Exp. Biol. 2009, 212, 1990-1994.

(57) Liu, D.; Tarakanova, A.; Hsu, C. C.; Yu, M.; Zheng, S.; Yu, L.; Liu, J.; He, Y.; Dunstan, D. J.; Buehler, M. J. Spider dragline silk as torsional actuator driven by humidity. Sci. Adv. 2019, 5, No. eaau9183.

(58) Tschoegl, N. W.; Knauss, W. G.; Emri, I. Poisson's ratio in linear viscoelasticity-a critical review. Mech. Time-Depend. Mater. 2002, 6, 3-51.

(59) Greaves, G. N.; Greer, A. L.; Lakes, R. S.; Rouxel, T. Poisson's ratio and modern materials. Nat. Mater. 2011, 10, 823-837.

(60) Yazawa, K.; Malay, A. D.; Masunaga, H.; Numata, K. Role of skin layers on mechanical properties and supercontraction of spider dragline silk fiber. Macromol. Biosci. 2019, 19, No. 1970006.

(61) Wu, P. J.; Kabakova, I. V.; Ruberti, J. W.; Sherwood, J. M.; Dunlop, I. E.; Paterson, C.; Török, P.; Overby, D. R. Water content, not stiffness, dominates Brillouin spectroscopy measurements in hydrated materials. Nat. Methods 2018, 15, 561.

(62) Scarcelli, G.; Polacheck, W. J.; Nia, H. T.; Patel, K.; Grodzinsky, A. J.; Kamm, R. D.; Yun, S. H. Noncontact three-dimensional mapping of intracellular hydromechanical properties by Brillouin microscopy. Nat. Methods 2015, 12, 1132.

(63) Zemlin, C. A Study of the Mechanical Behavior of Spider Silks, Technical Report TR69-29-CM (AD684333); US Army Natick Laboratories: Natick, MA, 1968; pp 13-30.

(64) Keten, S.; Xu, Z.; Ihle, B.; Buehler, M. J. Nanoconfinement controls stiffness, strength and mechanical toughness of $\beta$-sheet crystals in silk. Nat. Mater. 2010, 9, 359. 